\title{
Necrosis colónica secundaria. Informe de caso
}

\author{
Secondary colonic necrosis. Case report
}

$M^{a}$ del Carmen Aburto-Fernández', Adán Araujo-López ${ }^{*}$, Irwin U. García-Godoy', Antonio AlvaradoGonzález², José L. Gutiérrez-Samperio², Alfonso Álvarez-Manila de Orendáin², Mario A. Romero Ocio², Luis R. Arteaga-Villalba ${ }^{2}$, Arturo Herrera-Díaz', Christian O. Jiménez-Ríos ${ }^{1}$ y Ricardo M. Lerma-Alvarado ${ }^{1}$ ${ }^{1}$ Servicio de Cirugía General; '2Servicio de Cirugía de Colon y Recto. Hospital General de Querétaro, Querétaro, México

\section{Resumen}

Antecedentes: La lesión vascular colónica secundaria a la sobredosis de verapamilo, es mediada por radicales libres, formando microtrombos vasculares y generación de endotoxinas, siendo un diagnostico difícil. Caso Clínico: Femenino de 27 años, ingresa con abdomen agudo de 4 días posteriores a un evento remitido de intento suicida por sobredosis de verapamilo, interviniéndose quirúrgicamente donde se halla necrosis colónica transmural derecha, realizando hemicolectomía derecha con ileostomía terminal. Conclusiones: Reconocer y tratar de forma adecuada un abdomen agudo, no siempre se suele llegar a un adecuado diagnostico, por lo cual una minuciosa anamnesis lograría concluirlo.

PALABRAS CLAVE: Vascularidad colónica. Verapamilo. Abdomen agudo.

\begin{abstract}
Background: Colonic vascular lesion secondary to verapamil overdose is mediated by free radicals, forming vascular microtrombos and endotoxin generation, being a difficult diagnosis. Clinical Case: A 27-year-old female is admitted with an acute abdomen of 4 days after an event referred for a suicidal attempt due to an overdose of verapamil, operating surgically where there is a right transmural colon necrosis, performing a right hemicolectomy with terminal ileostomy. Conclusions: Recognize and properly treat an acute abdomen, not always reach an adequate diagnosis, so a thorough history could conclude.
\end{abstract}

KEY WORDS: Colonic vascularity. Verapamil. Acute abdomen.

\section{Introducción}

Los bloqueadores de los canales de calcio (BCC) están indicados en el tratamiento de la hipertensión arterial, angina estable, arritmias, migraña, fenómeno de Raynaud e incluso como tratamiento profiláctico de la hemorragia subaracnoidea'.

Dentro de los efectos adversos más frecuentes figuran hipotensión, bradiarritmias e isquemia intestinal, con cierta proclividad por el sistema vascular mesentérico, en especial la arteria mesentérica superior ${ }^{1,2}$.

El verapamilo posee efecto dual de acuerdo con su dosis; sus valores bajos o terapéuticos son de $5 \mathrm{mg} /$ dosis y puede administrarse en operaciones de isquemia intestinal, para disminuir en cierto grado la endotoxemia; empero, en los casos por sobredosis, el efecto es opuesto y provoca daño en la microcirculación y necrosis intestinal1-3.

\author{
Correspondencia: \\ *Adán Araujo-López \\ Circuito Andamaxei, 6 \\ Col. Paseos del Bosque \\ C.P. 76910, Corregidora, Querétaro, México \\ E-mail: draraujolopez@gmail.com.
}

Fecha de recepción: 10-08-2018

Fecha de aceptación: 15-12-2018

DOI: $10.24875 / C I R U .18000656$
Cir Cir. 2019;87(S1):33-37

Contents available at PubMed www.cirugiaycirujanos.com 
La isquemia intestinal secundaria a intoxicación por verapamilo se presenta a dosis $>0.5-1 \mathrm{mg} / \mathrm{kg} / \mathrm{dosis}^{4,5}$. Debido a su propensión por la arteria mesentérica superior, sobre todo por su primera rama (cólica derecha), la intoxicación con verapamilo predispone al desarrollo de isquemia-necrosis del hemicolon derecho ${ }^{5}$.

\section{Caso clínico}

Paciente femenina de 27 años con antecedentes importante de dispositivo intrauterino desde ocho años antes, que ingresa al servicio de urgencias dos horas después de la ingestión de 20 pastillas de verapamilo como intento de suicidio. El cuadro clínico incluye hipotensión, taquicardia, obnubilación, taquipnea, signos vitales expresados con tensión arterial de 70/53 (no requirió tratamiento con aminas), frecuencia cardiaca (101 x'), saturación de oxígeno (88 \%), electrocardiograma con ritmo sinusal y gasometría con acidosis metabólica compensada; la intoxicación del efecto se contrarrestó con lavado de carbón activado (dosis de $1 \mathrm{~g} / \mathrm{kg}$ ), se extrajeron restos de pastillas y se administraron gluconato de calcio y soluciones cristaloides para mejorar la tensión arterial. Ante una mejoría de 24 horas posteriores al episodio de intoxicación por intento de suicidio, se decidió egresar a la paciente: consciente, orientada en sus tres esferas, con tensión arterial de 110/73 mmHg, sin taquicardia, en deambulación, sin náusea o vómito ni dolor abdominal o precordial; tampoco hay fosfenos ni cefalea, tolera dieta completa y cumple los criterios de egreso hospitalario. Recibe el alta domiciliaria con vigilancia médica y se programa para atención ambulatoria con el servicio de psiquiatría.

Reingresa a urgencias cuatro días después, con abdomen agudo (a expensas de silencio abdominal, hiperestesia, hiperbaralgesia y resistencia muscular involuntaria), con evacuaciones líquidas fétidas, verdosas, flujo vaginal espeso y fétido, con grumos, además de náusea y vómito constante de características intestinales; fiebre de $39^{\circ} \mathrm{C}$, leucocitosis de 26000 , neutrofilia de $93 \%$ y PCR de $36 \mathrm{mg} / \mathrm{dl}$.

Los signos vitales al ingreso fueron los siguientes: tensión arterial (TA), 128/70 mmHg; frecuencia cardiaca (FC), 130 Ipm; saturación de $\mathrm{O}_{2}, 90 \%$; la TC abdominal revela oclusión intestinal sin zona de transición, ni visualización de colon derecho, con abundante líquido libre en cavidad pélvica, útero edematizado e irregular. Ante los hallazgos clínicos y tomográficos se decide intervenir mediante una laparotomía exploradora que obtiene $1000 \mathrm{ml}$ de líquido libre purulento, abscesos pélvico y en corredera parietocólica derecha, con necrosis abscedada sobre el territorio de la arteria cólica derecha (Figs. 1 y 2); (íleon terminal, colon ascendente, ángulo hepático, mitad del colon transverso), por lo cual se practica hemicolectomía derecha extendida, con cierre en bolsa de Hartman del transverso e ileostomía terminal (Fig. 3).

\section{Histopatología del segmento de colon}

Infarto hemorrágico, consecutivo a trombosis vascular, sin datos de malignidad, con colitis crónica y aguda grave adjunta (Fig. 4). El postoperatorio cursa con estabilidad, tratamiento antisecretor por alto gasto de ileostomía $>10 \mathrm{ml} / \mathrm{kg} / 24 \mathrm{~h}$. A los cinco días inician elevaciones febriles y hay gasto por drenaje de tipo abierto seropurulento; la TC abdominal revela una colección en la corredera parietocólica derecha y pélvica, por lo cual se decide explorar y drenar de forma quirúrgica; se identifica un abdomen sensible, se drenan las dos colecciones de $100 \mathrm{~cm}^{3}$, se cierra el abdomen sin complicaciones y se reinicia el tratamiento antisecretor, con medidas astringentes por gastos altos de ileostomía, que se conserva; la paciente puede egresar controlada y hoy día se halla en protocolo de restitución intestinal.

\section{Discusión}

Los BCC se introdujeron en 1960 para las evaluaciones cardiovasculares. Los efectos secundarios más frecuentes de los BBC son hipotensión y bradiarritmias, así como complicaciones isquémicas, entre ellas necrosis digital o de una extremidad, necrosis tubular aguda, isquemia miocárdica, necrosis tubular aguda, isquemia intestinal, o hemorragia gastrointestinal $(6 \%)^{1,3}$. Las indicaciones médicas de los BCC incluyen hipertensión, angina estable, arritmias, migraña, fenómeno de Raynaud y hemorragia subaracnoidea'.

Algunos de los tratamientos de la intoxicación por BCC en general, y que han mostrado mejoría en su evolución, son el calcio intravenoso (IV), los vasopresores (norepinefrina, 40\%), la dopamina (27\%), la epinefrina (27\%), el isoproterenol (15\%), la dobutamina $(6.25 \%)$ y el glucagón (infusión o bolos a dosis media de $2 \mathrm{~g})^{1}$.

La colitis isquémica representa un cuadro de isquemia mesentérica no oclusiva, efecto de la reducción de la perfusión colónica, y es un hallazgo transoperatorio de presentación infrecuente ${ }^{1,3}$. El colon 


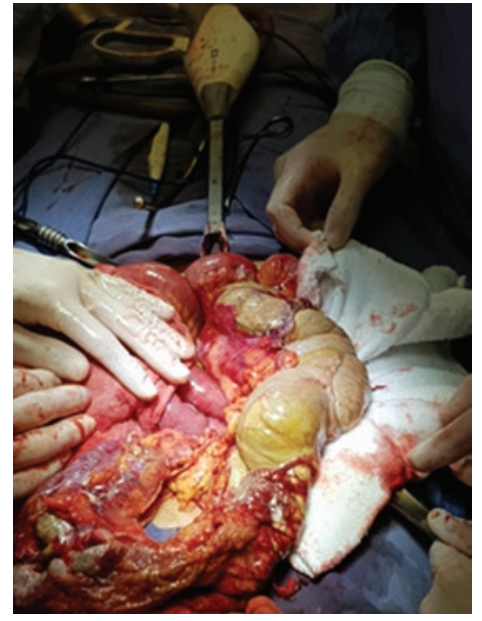

Figura 1. Isquemia intestinal abscedada sobre el territorio de la cólica derecha (marzo de 2018).

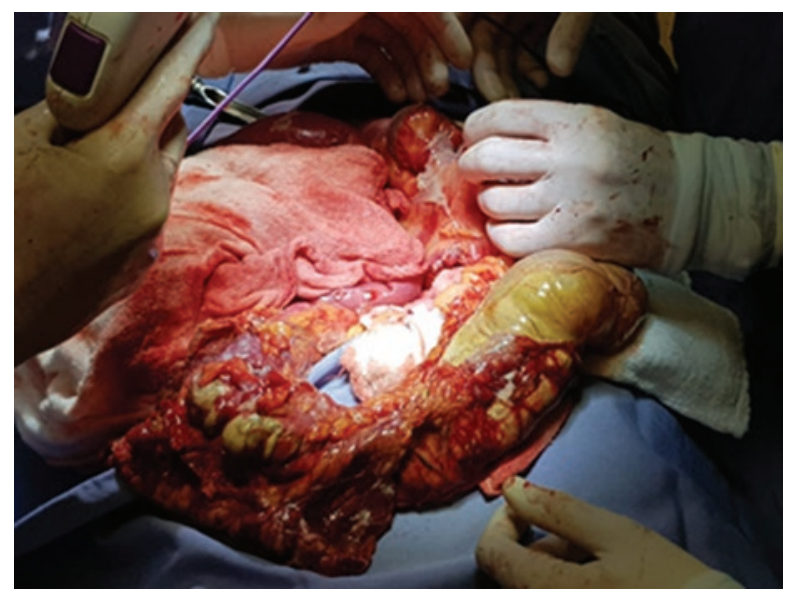

Figura 2. Colon ascendente y ángulo hepático comprometido por isquemia, necrosis e infección.

izquierdo está comprometido con mayor frecuencia, pero el cuadro de isquemia intestinal aguda puede afectar al territorio de la arteria mesentérica superior de forma predominante sobre la cólica derecha².

La colitis isquémica se clasifica en dos tipos: el tipo I es una colitis espontánea sin identificación de factor causal y afecta cualquier parte del colon; tiene predominio izquierdo, y puede desarrollar necrosis y perforación en 15 a 25\% (en $82 \%$ de esta última cifra se requiere intervención quirúrgica²); el tipo II tiene una causa secundaria, más a menudo un episodio de hipotensión sistémica o decremento del gasto cardiaco o después de una operación aortovascular ${ }^{2,3}$.

La histopatología identifica con más frecuencia necrosis e inflamación extendida, y transmural, con edema de submucosa ${ }^{2}$. El tratamiento por intoxicación de antagonistas del calcio incluye la descontaminación gastrointestinal, calcio intravenoso e hiperinsulinoterapia euglucémica para controlar la hipotensión ${ }^{3}$.

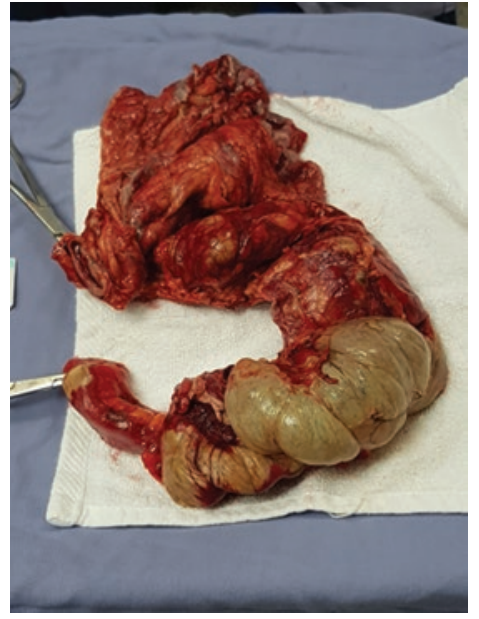

Figura 3. Hemicolectomía derecha extendida sobre tejido localizado comprometido en extensión transmural.

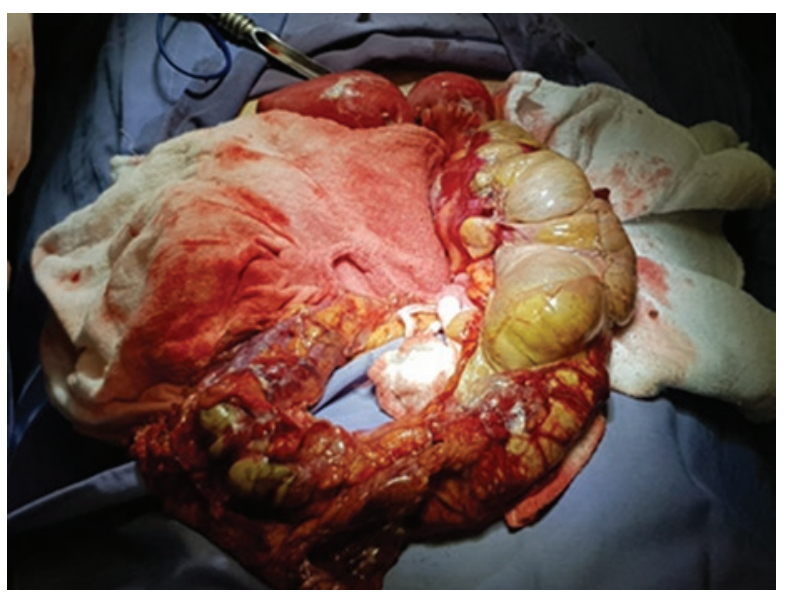

Figura 4. Pieza patológica con necrosis circunscrita en el territorio de la cólica derecha; compromiso de la válvula ileocecal.

Entre los hallazgos transquirúrgicos por medio de laparotomías se han descrito infartos intestinales extensos, con predominio de colon ${ }^{4}$.

La perfusión intestinal tiene una relación con el factor activador plaquetario (PAF) y existe una proclividad por el mesenterio de la arteria mesentérica superior. En el plano fisiopatológico, dado que hay isquemia intestinal, se desarrolla una endotoxemia, en la cual el índice de neutrófilos estimula a las células de Kupffer que en el hígado liberan citocinas proinflamatorias como IL- 6 y TNF- $\alpha$ y radicales libres, relacionados con la disfunción microvascular y del parénquima intestinal5, ${ }^{5,6}$.

Dentro de los fármacos que han promovido la protección durante una endotoxemia por isquemia intestinal se ha demostrado la participación del propofol, así como del verapamilo, si bien como bolo, 10 minutos antes de la intervención; sin embargo, su toxicidad radica en su afinidad celular al inhibir la xantina 
oxidasa y esperar al calcio mitocondrial, o por efectos secundarios como la supresión de la producción de radicales libres y citocinas proinflamatorias por las células de Kupffer ${ }^{6}$.

Gundes, et al., realizaron en el 2013 un estudio en el que demostraron que la causa que puede contribuir a la isquemia intestinal secundaria es la aterosclerosis (con sus principales factores: hipertensión arterial [6\%] y enfermedad renal crónica [5\%], con mayor riesgo si existe tratamiento sustitutivo con hemodiálisis por hipotensión). Una presentación común es efecto del bajo flujo sanguíneo territorial, cuya consecuencia es el dolor abdominal quirúrgico en un $13 \%$ (semejante a una apendicitis aguda), con isquemia intestinal secundaria; el tratamiento ideal es la hemicolectomía derecha con anastomosis primaria ${ }^{4-5}$ debido a que la isquemia puede estar localizada ${ }^{7}$ en $56 \%$.

El diagnóstico de la isquemia intestinal de colon es difícil, por lo cual deben considerarse los antecedentes. Uno de los trastornos que más se asemejan a la isquemia cecal (la forma más común de lesión isquemia gastrointestinal) es la apendicitis, cuyo diagnóstico se establece hasta en $36 \%$ de manera transoperatoria ${ }^{8,9}$.

Ante este índice diagnóstico, los estudios de imagen muestran pocos hallazgos: adelgazamiento de la pared del colon (abscesos) y flujo vascular reducido; más aún, Ripolles, et al., describieron que la alteración de la grasa pericólica puede ser el único hallazgo $0^{8,9}$.

Perbert, et al., notificaron en 2008 un caso de intoxicación por verapamilo por intento suicida y en él se describe que la isquemia mesentérica de colon derecho es de tipo no oclusivo ${ }^{10}$. Lee, et al., publicaron en el 2010 el tercer caso de intoxicación por bloqueadores de los canales de calcio (nifedipina), en este paciente después del lavado gástrico y con desarrollo de isquemia intestinal de colon derecho de tipo no oclusiva; en este cuadro se presentaron abscesos transmurales de forma similar al caso descrito aquí $^{11}$.

\section{Conclusiones}

La isquemia intestinal secundaria es un tema amplio; sin embargo, al enfocarse en este caso de una paciente que sufrió una intoxicación secundaria a verapamilo por intento suicida, se ha mostrado una nueva ventana para corroborar de forma médica la propensión de los bloqueadores de los canales de calcio por la arteria mesentérica superior, sobre todo en su primera rama, la cólica derecha. Se ha demostrado que ante un episodio de hipotensión y bajo gasto sostenido, por intoxicación de BCC, los radicales libres y la microcirculación entérica se comprometen. En consecuencia, si al ingreso hay hipotensión con tensión arterial media $<63 \mathrm{mmHg}$, además de sospecha clínica, por más leve que sea, es preciso indagar y tratar de inmediato, ya que la aparición de bradiarritmias, cefalea y dolor abdominal podría contribuir a complicar la evolución posterior en el egreso domiciliario.

Esto favorecería por tanto un isquemia intestinal secundaria; sin embargo, si se identifica de modo adecuado un episodio de hipotensión secundaria, ya sea por BCC o cualquier otro antihipertensivos, puede evitarse este tipo de complicaciones.

\section{Conflicto de intereses}

Los autores declaran que no tienen ningún conflicto de intereses.

\section{Financiamiento}

Para la realización de este artículo no se recibió patrocinio.

\section{Agradecimientos}

Los autores agradecen la atención integral y adecuada del paciente coordinada en todos sus aspectos desde el Servicio de Urgencias, y los residentes y médicos adscritos de los servicios de Cirugía General, Colon y Recto, Anestesiología, Psiquiatría, Nutrición Clínica, Enfermería Quirúrgica y General del Hospital General de Querétaro.

Asimismo, agradecemos a esta paciente, que nos dio su confianza y que se retrajo del suicidio, y que pudo recibir el alta con una notable mejoría física, psicológica, nutricional y médica.

\section{Responsabilidades éticas}

Protección de personas y animales. Los autores declaran que para esta investigación no se han realizado experimentos en seres humanos ni en animales.

Confidencialidad de los datos. Los autores declaran que han seguido los protocolos de su centro de trabajo sobre la publicación de datos de pacientes. 
Derecho a la privacidad y consentimiento informado. Los autores han obtenido el consentimiento informado de los pacientes y/o sujetos referidos en el artículo. Este documento obra en poder del autor de correspondencia.

\section{Bibliografía}

1. Levine M, Curry S, Padilla-Jones A, Ruha A. Critical care management of verapamil and diltiazem overdose with a focus on vasopressors: a 25-year experience at a single center. Toxicology/Original Research. Ann Emer Med. 201362(3). Disponible en: https://www.ncbi.nlm.nih.gov/pubmed/23642908

2. Schuler J, Margaret M, Hudlin HD. Cecal necrosis infrequent variant of ischemic colitis. Dis Colon Rectum. 2000;43(5):708-15. Disponible en https://link.springer.com/article/10.1007\%2FBF02235593.

3. Devasahayam J, Pillal I, Uppaluri. Acute severe intestinal obstruction secondary to amlodipine toxicity. Q J Med. 2011;1(3):1-3. Disponible en: https://academic.oup.com/qjmed/article/105/5/467/1564727.

4. Chan T, Chan Y, Lua F. Ischemia complications after calcium channel blocker poisoning. Ann Emer Med 2014;63 (1):1. Disponible en: https:// www.annemergmed.com/article/S0196-0644(13)01263-8/fulltext.
5. Hsueh W, González-Crussi F, Arroyave J. Platelet-activating factor-induced ischemic bowel necrosis. AJP. 1986;122(2). Disponible en: https:// www.nature.com/articles/pr1993772.

6. Kaplan N, Yagmurdur H, Kilinc K, Baltaci B, Tezel S. The protective effects of intravenous anesthetics and verapamil in gut ischemia/reperfusion-induced liver injury. 2007;105(5):1371-79. Disponible en: https:// insights. ovid.com/pubmed?pmid=17959968.

7. Gundes E, Kucukkartallar T, Haldun M, Cakir M, Aksoy F. Ischemic necrosis of the cecum: a single center experience. Korean J Gastroenterol. 2013;61(5);265-269. Disponible en: https://synapse.koreamed.org/ Synapse/Data/PDFData/0028KJG/kjg-61-265.pdf.

8. Watanabe T, Tomita S, Shirane H, Yoshihiro O, Orino A, Akio T, et al. Cecal necrosis due to ischemic colitis mimicking an abscess on sonography. J Ultra Med 2006;25(5): 393-396. Disponible en: https://doi. org/10.7863/jum.2006.25.3.393.

9. Gutiérrez $\mathrm{H}$, Jorgensen $\mathrm{M}$. Colonic ischemia after verapamil overdose. Ann Inter Med 1996;124(1)535-36. Disponible en: https://sci-hub. tw/10.7326/0003-4819-124-5-199603010-00020.

10. Perbet $S$, Constantin J-M, Guèrin R, Faure $M$, Brugére $C$, Inés $D$, et al. Non-occlusive colonic ischemia induced by verapamil ER overdose. Intensive Care Med. 2009;35:956-957. Disponible en: https://sci-hub. tw/10.1007/s00134-008-1375-2.

11. Lee $Y$, Chan $Y$, Lau F. Extensive bowel ischaemia after nifedipine overdose. Hong Kong J Emer Med. 2010;17(4):361-365. Disponible en: https://hkcem.com/html/publications/Journal/2010-4/p360-363.pdf. 